\title{
Inventario de Evaluación de la Personalidad para adolescentes: consistencia interna y dimensionalidad en adolescentes de Buenos Aires, Argentina
}

Juliana Beatriz Stover ${ }^{\star}$, Guadalupe de la Iglesia ${ }^{\star *}$, Alejandro Castro-Solano ${ }^{\star \star \star}$, Mercedes $^{*}$ Fernández-Liporace ${ }^{\star * * *}$

* Doctora en Psicología. Investigadora asistente, Consejo Nacional de Investigaciones Científicas y Técnicas; Facultad de Psicología, Universidad de Buenos Aires. Jefa de trabajos prácticos regular, Facultad de Psicología, Universidad de Buenos Aires, Argentina. Correo electrónico: julianastover@psi.uba.ar

** Doctora en Psicología. Investigadora asistente, Consejo Nacional de Investigaciones Científicas y Técnicas; Facultad de Psicología, Universidad de Buenos Aires. Jefa de trabajos prácticos regular, Facultad de Psicología, Universidad de Buenos Aires, Argentina. Correo electrónico: gdelaiglesia@gmail.com

*** Doctor en Psicología. Investigador principal, Consejo Nacional de Investigaciones Científicas y Técnicas; Departamento de Psicología, Facultad de Ciencias Sociales, Universidad de Palermo. Profesor adjunto regular, Facultad de Psicología, Universidad de Buenos Aires, Argentina. Correo electrónico: acastro1@palermo.edu

**** Doctora en Psicología. Investigadora principal, Consejo Nacional de Investigaciones Científicas y Técnicas; Facultad de Psicología, Universidad de Buenos Aires. Profesora titular regular, Facultad de Psicología, Universidad de Buenos Aires, Argentina. Correo electrónico:mliporac@psi.uba.ar

Recibido: 29 de noviembre del 2016

Aprobado: 12 de junio del 2017

Cómo citar este artículo: Stover, J. B., De la Iglesia, G., Castro-Solano, A. y FernándezLiporace, M. (2017). Inventario de Evaluación de la Personalidad para adolescentes: consistencia interna y dimensionalidad en adolescentes de Buenos Aires, Argentina. Pensando Psicología, 13(22), 15-27. doi: https://doi.org/10.16925/pe.v13i22.1985

\section{Resumen}

Introducción: el Inventario de Evaluación de la Personalidad para adolescentes (PAI-A, por sus siglas en inglés) examina la sintomatología psicopatológica y las variables vinculadas al diseño de intervenciones psicoterapéuticas. Consta de 264 ítems para su uso en la evaluación clínica de adolescentes entre 12 y 18 años. Incluye escalas de validez clínicas, relacionadas con el tratamiento y de relación interpersonal. Objetivo: en este trabajo se presentan análisis psicométricos del Inventario de Evaluación de la Personalidad para adolescentes. Método: Participaron 1.002 adolescentes (50,3\% mujeres, 49,7\% varones; $\left.\mathrm{M}_{\text {edad }}=14,99 ; \mathrm{DE}=1,88\right)$, residentes en la ciudad de Buenos Aires y alrededores, en Argentina. Se calcularon alfas de Cronbach para estimar la consistencia interna, y se advirtieron valores excelentes para las escalas clínicas, relacionadas con el tratamiento y de relación interpersonal, y aceptables para las escalas de validez y subescalas. Resultados: se examinó la dimensionalidad mediante análisis de componentes principales con rotación Varimax. Se aisló una estructura de cuatro factores al incluir las 22 escalas, mientras que al analizar las 11 clínicas, se obtuvieron dos factores. Conclusiones: como conclusión, se aportan evidencias de la calidad psicométrica del PAI-A para su uso con adolescentes de Buenos Aires, cubriendo un área de vacancia en la evaluación psicológica local. Futuras investigaciones deberán ampliar esos análisis de calidad psicométrica, así como replicar los procedimientos en muestras clínicas.

Palabras clave: adolescentes, consistencia interna, dimensionalidad, PAI-A. 


\title{
Personality Assessment Inventory for Adolescents: Internal Consistency and Dimensionality in Adolescents from Buenos Aires, Argentina
}

\begin{abstract}
Introduction: The Personality Assessment Inventory for Adolescents (PAI-A) examines the psychopathological symptomatology and variables related to the design of psychotherapeutic interventions. It is composed of 264 items for use in the clinical assessment of adolescents between 12-18 years old. Validity, clinical, treatment-related, and interpersonal relationship scales are included. Purpose: This paper presents psychometric analyses of the Personality Assessment Inventory for Adolescents. Method: The participants were 1,002 adolescents (50.3\% women, $49.7 \%$ men, $\mathrm{M}_{\text {age }}=14.99, \mathrm{SD}=1.88$ ) living in Buenos Aires (Argentina) and on the outskirts. Cronbach's alphas were calculated to estimate internal consistency, with excellent values for clinical, treatment-related and interpersonal relationship scales, and acceptable values for validity scales and subscales. Results: Dimensionality was examined by principal component analysis with a varimax rotation. A four-factor structure was isolated by including the 22 scales, whereas two factors were obtained when analyzing the 11 clinical scales. Conclusions: We provide evidence of the psychometric quality of the PAI-A for use with adolescents from Buenos Aires, filling a gap in local psychological assessment. Further research should extend these psychometric quality analyses, and replicate the procedures in clinical samples.
\end{abstract}

Keywords: adolescents, internal consistency, dimensionality, PAI-A.

\section{Inventário de Avaliação da Personalidade para adolescentes: consistência interna e dimensionalidade em adolescentes de Buenos Aires}

\section{Resumo}

Introdução: o Inventário de Avaliação da Personalidade para adolescentes (PAI-A, por sua sigla em inglês) examina a sintomatologia psicopatológica e as variáveis vinculadas ao desenho de intervenções psicoterapéuticas. Consta de 264 itens para seu uso na avaliação clínica de adolescentes entre 12 e 18 anos. Inclui escalas de validade, clínicas, relacionadas com o tratamento e de relação interpessoal. Objetivo: neste trabalho, apresentam-se análises psicométricas do PAI-A. Método: participaram 1002 adolescentes (50,3\% mulheres, 49,7\% homens; $M_{\text {idade }}=14,99 ; \mathrm{DE}=1,88$ ), residentes na cidade de Buenos Aires e municípios vizinhos. Calcularam-se alfas de Cronbach para estimar a consistência interna, advertendo-se valores excelentes para as escalas clínicas, relacionadas com o tratamento e de relação interpessoal, e aceitáveis para as escalas de validade e subescalas. Resultados: examinou-se a dimensionalidade mediante análise de componentes principais com rotação Varimax. Isolou-se uma estrutura de quatro fatores ao incluir as 22 escalas, enquanto, ao analisar as 11 clínicas, foram obtidos dois fatores. Conclusões: como conclusão, contribuem-se com evidências da qualidade psicométrica do PAI-A para seu uso com adolescentes de Buenos Aires, preenchendo um vazio na avaliação psicológica local. Futuras pesquisas deverão ampliar essas análises de qualidade psicométrica bem como reproduzir os procedimentos em amostras clínicas.

Palavras-chave: adolescentes, consistência interna, dimensionalidade, PAI-A. 


\section{Introducción}

La adolescencia constituye una etapa de cambios físicos, psíquicos y sociales en la que los sujetos se hallan con mayor riesgo de padecer trastornos mentales (Casullo y Fernández Liporace, 2001; Vega et al., 2009). Por ejemplo, según la Organización Mundial de la Salud (oms, 2014), el suicidio se ubica en tercer lugar como causa de muerte en adolescentes y la depresión se erige como el principal motivo de enfermedad y discapacidad, sumándose en octavo lugar los trastornos de ansiedad. A su vez, la psicopatología en adolescentes se vincula con problemas en el funcionamiento social, el rendimiento académico y la sintomatología presente en la adultez (Helgeland, Kjelsberg y Torgersen, 2005; Quiroga et al., 2013; Stepp et al., 2013). Por lo tanto, una correcta evaluación de la sintomatología psicopatológica en este grupo etario resulta crucial de cara a la correcta detección y tratamiento de sujetos con padecimientos psicológicos. Esto permitirá un diagnóstico y su consecuente atención psicológica, la cual repercutirá, eventualmente, en su funcionamiento psicosocial actual y futuro.

Entre los instrumentos disponibles para examinar la psicopatología de adolescentes de Buenos Aires (Argentina) se encuentran técnicas de diagnóstico como el Inventario Multifásico de la Personalidad en su versión para adolescentes (MMPI-A; Casullo, 2003) y el Inventario Clínico para Adolescentes de Millon (MACI; Casullo, Góngora y Castro, 1998), en las que se ha producido un envejecimiento de los baremos. Por otro lado, se encuentran instrumentos de rastrillaje como el Listado de Chequeo de Síntomas Revisado (sCL-90-R; Casullo y Pérez, 1998), que posee baremos construidos en el 2008 (Casullo y Pérez, 1998), pero que no cuenta con estudios de calidad psicométrica para población adolescente. En relación con este último aspecto, se han desarrollado escalas diseñadas específicamente para este grupo, pero que solo cubren facetas puntuales de la psicopatología como, por ejemplo, el riesgo suicida (Desuque, Rubilar y Lemos, 2011; Fernández Liporace y Casullo, 2006), problemas alimenticios (Góngora, 2010; Rutsztein et al., 2013), estrés (Oros y Neifert, 2006) y dificultades atencionales (Grañana et al., 2011), entre otros. Ante este panorama, contar con instrumentos que analicen un amplio espectro de variables psicopatológicas en adolescentes locales surge claramente como área de vacancia.

El Inventario de Evaluación de la Personalidad para adolescentes (PAI-A; Morey, 2007a) se presenta como una opción de interés para llenar este vacío. Fue diseñado como complemento de la forma para adultos
(PAI; Morey, 1991 y 2007b), modificando los reactivos para que se adecuaran a las características de la población objetivo, así como reduciendo el número de ítems. Al igual que en la versión para adultos, su finalidad es examinar sintomatología psicopatológica y variables vinculadas al diseño de intervenciones psicoterapéuticas. Consta de 264 ítems para su uso en la evaluación clínica de adolescentes entre 12 y 18 años. Sus reactivos prevén una respuesta con formato Likert de cuatro posiciones (falsas, ligeramente verdaderas, bastante verdaderas, completamente verdaderas), lo cual permite captar una mayor variabilidad en la intensidad o gravedad de las manifestaciones. Incluye escalas de validez, clínicas, relacionadas con el tratamiento y de relación interpersonal. Las cuatro escalas de validez evalúan respondiendo al objetivo de captar distintos sesgos y/o intenciones en la actitud de respuesta del examinado. Las 11 escalas clínicas constan, en su mayoría, de subescalas destinadas a ampliar la información sobre cada caso. En la tabla 1 , se detallan los nombres de escalas y subescalas.

La versión para adultos del PAI se presenta como un instrumento adecuado para evaluar varios trastornos según los criterios propuestos por el DSM-5 (American Psychiatric Association, 2013; Busch, Morey y Hopwood, 2017; Hopwood et al., 2013), a lo que se suman recientes trabajos que han utilizado el PAI-A demostrando su utilidad tanto para el trabajo clínico (Morey y Meyer, 2014; Turner, 2014), como para el ámbito de investigación (Ho, Cheung y Cheung, 2008; Ríos y Morey, 2013).

Los estudios psicométricos realizados con población adolescente son escasos y predominan los desarrollados para versión para adultos, lo que resulta en que las adaptaciones en torno al PAI-A siguen siendo un área en desarrollo (Morey y Meyer, 2013). Se han efectuado adaptaciones en Estados Unidos (Morey, 2007a), España (Cardenal, Ortiz-Tallo y Santamaría, 2012) y China (Cheung et al., 2008); sin embargo, los estudios psicométricos con base en tales versiones han sido poco promovidos.

En relación con la consistencia interna del PAIA, se han reportado valores excelentes para las escalas y más bajos para las subescalas y escalas de validez tanto en la versión para adolescentes desarrollada en Estados Unidos (Morey, 2007a), como en su adaptación china (Cheung et al., 2008). Respecto al PAI, se han presentado hallazgos similares para la forma original estadounidense (Morey, 1991 y 2007b) y las adaptaciones alemana (Groves y Engel, 2007), griega (Lyrakos, 2011) y española (Ortiz-Tallo et al., 2011). 
Tabla 1

PAI-A: escalas y subescalas

\begin{tabular}{|c|c|c|}
\hline \multirow{4}{*}{ Validez } & Inconsistencia (INC) & - \\
\hline & Infrecuencia (INF) & - \\
\hline & Impresión negativa (IMN) & - \\
\hline & Impresión positiva (IMP) & - \\
\hline \multirow{30}{*}{ Clínicas } & & Conversión (sOM-c) \\
\hline & Quejas somáticas (som) & Somatización (som-s) \\
\hline & & Hipocondría (som-H) \\
\hline & & Cognitiva (ANS-C) \\
\hline & Ansiedad (ANs) & Emocional (ANS-E) \\
\hline & & Fisiológica (ANS-F) \\
\hline & & Obsesivo-compulsivo (TRA-O) \\
\hline & Trastornos relacionados con la ansiedad (TRA) & Fobias (TRA-F) \\
\hline & & Estrés postraumático (TRA-E) \\
\hline & & Cognitiva (DEP -C) \\
\hline & Depresión (DEP) & Emocional (DEP-E) \\
\hline & & Fisiológica (DEP-F) \\
\hline & & Nivel de actividad (MAN-A) \\
\hline & Manía (MAN) & Grandiosidad (MAN-G) \\
\hline & & Irritabilidad (MAN-I) \\
\hline & & Hipervigilancia (PAR-H) \\
\hline & Paranoia (PAR) & Persecución (PAR-P) \\
\hline & & Resentimiento (PAR-R) \\
\hline & & Experiencias psicóticas (ESQ-P) \\
\hline & Esquizofrenia (ESQ) & Indiferencia social (ESQ-S) \\
\hline & & Alteración del pensamiento (ESQ-A) \\
\hline & & Inestabilidad emocional (LIM-E) \\
\hline & & Alteración de la identidad (LIM-I) \\
\hline & Rasgos límites (LIM) & $\begin{array}{l}\text { Relaciones interpersonales problemáticas } \\
\text { (LIM-P) }\end{array}$ \\
\hline & & Autoagresiones (LIM-A) \\
\hline & & Conductas antisociales (ANT-A) \\
\hline & Rasgos antisociales (ANT) & Egocentrismo (ANT-E) \\
\hline & & Búsqueda de sensaciones (ANT-B) \\
\hline & Problemas con el alcohol (ALC) & - \\
\hline & Problemas con las drogas (DRG) & - \\
\hline \multirow{7}{*}{$\begin{array}{l}\text { Vinculadas al } \\
\text { tratamiento }\end{array}$} & & Actitud agresiva (AGR-A) \\
\hline & Agresión (AGR) & Agresión verbal (AGR-V) \\
\hline & & Agresión física (AGR-F) \\
\hline & Ideación suicida (suI) & - \\
\hline & Estrés (EST) & - \\
\hline & Falta de apoyo social (FAs) & - \\
\hline & Rechazo al tratamiento (RTR) & - \\
\hline \multirow{2}{*}{$\begin{array}{c}\text { Relación } \\
\text { interpersonal }\end{array}$} & Dominancia (DOM) & - \\
\hline & Afabilidad (AFA) & - \\
\hline
\end{tabular}


La dimensionalidad del inventario ha sido frecuentemente analizada en el caso del PAI adultos, considerando las puntuaciones de las escalas como input, pero los estudios sobre el PAI-A resultan insuficientes. Esta línea de trabajo comenzó con la versión original del PAI (Morey, 1991 y 2007b), con la que se efectuó un análisis de componentes principales con rotación Varimax incluyendo las 11 escalas clínicas, que se agruparon en dos factores que explicaron el 77,20\% de la varianza. En la versión alemana (Groves y Engel, 2007), se replicó el procedimiento y se hallaron nuevamente dos dimensiones que explicaron el 61,69\% de la varianza; y lo mismo sucedió en la adaptación española (Ortiz-Tallo et al., 2011), en la que una solución bifactorial dio cuenta del $68 \%$ de la varianza. A su vez, los investigadores alemanes y españoles también llevaron a cabo un segundo análisis, en el que se incluyeron las 22 puntuaciones de todas las escalas (validez, clínicas, relacionadas con el tratamiento y de relación interpersonal). En el primer caso, se halló una estructura que explicó el 62,37\% de la varianza a través de cuatro factores, mientras que en el segundo caso se aislaron cinco dimensiones que explicaron el $62 \%$ de la varianza. La estructura del PAI-A se analizó siguiendo estos procedimientos solamente en la adaptación china, en la que calculando un análisis de componentes principales con rotación Promax-se identificaron dos factores para las escalas clínicas (49,93\% de varianza explicada) y cuatro al considerar todas las escalas (Cheung et al., 2008).

En cuanto al análisis de los resultados obtenidos mediante el test, si bien no se basan en los estudios de dimensionalidad mencionados, Blais, Baity y Hopwood (2011) sugieren un agrupamiento para interpretar las escalas del PAI-A en función de la sintomatología que suelen presentan los adolescentes. En un primer grupo, se incluyen los síntomas internalizantes, como los problemas somatomorfos (SOM), de ansiedad (ANS) y manifestaciones disfóricas (DEP). También recomiendan considerar las escalas relacionadas con el tratamiento de ideación suicida y estrés.

En un segundo grupo, proponen contemplar aquellos patrones externalizantes, como las manifestaciones impulsivas e irritables (MAN), los desórdenes de conductas y los vínculos interpersonales en los que se ejerce explotación (ANT), así como problemas con el alcohol (ALC) y las drogas (DRG). Como complemento, se recomienda examinar la agresión. En el tercer agrupamiento, reúnen aquellos problemas del pensamiento, como manifestaciones paranoides (PAR) y estados confusionales (ESQ).
Por último, en el cuarto grupo incluyen alteraciones en los afectos, la autoimagen y las relaciones interpersonales (LIM), así como la falta de apoyo social (FAs) asociada a esos problemas. Las escalas de relación interpersonal, dominancia y afabilidad se consideran relevantes para todos los grupos, dada su aplicabilidad en todas las esferas de la vida del individuo.

Por todo lo hasta aquí expuesto, debido a la importancia de adaptar instrumentos que resulten adecuados para su uso en la población objetivo (Hambleton y Zenisky, 2011; Muñiz, Elosua y Hambleton, 2013) y en virtud de la referida escasez de escalas para examinar sintomatología psicológica en adolescentes de Buenos Aires, siguiendo investigaciones previas (Groves y Engel, 2007; Morey, 2007b; Ortiz-Tallo et al., 2011), los objetivos de este trabajo apuntan a: i) examinar la consistencia interna de las escalas y subescalas; y ii) analizar la dimensionalidad del PAI-A.

\section{Método}

Se trabajó con un diseño no experimental transversal y con un estudio descriptivo y explicativo. Los datos se recolectaron en población general no consultante mediante un muestreo intencional, estratificando según edad y sexo.

Participaron 1002 adolescentes (50,3\% mujeres, $49,7 \%$ varones), con edades comprendidas entre 12 y 18 años $(M=14,99 ; \mathrm{DE}=1,88)$. La totalidad de la muestra se encontraba escolarizada en instituciones de nivel medio $(60,4 \%$ en escuelas de gestión privada y el resto, en públicas).

\section{Instrumentos}

- Encuesta de datos sociodemográficos. Recolecta de información sobre características de los participantes, tales como sexo, edad y escolarización.

- Inventario de Evaluación de la Personalidad para adolescentes (Personality Assessment Inventory, PAI-A) (Morey, 2007a). Se empleó la versión española, sobre la cual se efectuaron adaptaciones lingüísticas. Consta de 264 ítems con opciones de respuesta Likert ( falso $=0$; levemente verdadero $=1$; bastante verdadero $=2$; completamente verdadero $=3$ ). Sus escalas y subescalas han sido descritas en la introducción de este trabajo. 


\section{Procedimiento}

A partir de la adaptación española del PAI-A, cinco jueces expertos en evaluación psicológica y psicometría revisaron los ítems, y examinaron su adecuación para su aplicación en el trabajo con adolescentes locales. Tras ello, se efectuó una administración piloto con 10 adolescentes, con el objetivo de poner a prueba la comprensión de los reactivos. Luego de efectuar modificaciones menores, se recogieron datos para la muestra de tipificación. Estudiantes avanzados de Psicología reclutaron participantes, coordinados por doctores en Psicología. Tanto padres como alumnos expresaron su conformidad con la toma de datos mediante un consentimiento informado, con lo que se garantizó la confidencialidad de la información. De este modo, se administraron 1.020 PAI-A durante el 2013. Se eliminaron los casos con gran cantidad de ítems sin responder o con datos sociodemográficos incompletos.

\section{Análisis}

Tras replicar los criterios utilizados en los trabajos de las versiones estadounidense, alemana y española del PAI (Groves y Engel, 2007; Morey, 2007b; Ortiz-Tallo et al., 2011), en un primer momento se calcularon coeficientes a de Cronbach para estimar la consistencia interna de las puntuaciones. Luego, con el objetivo de describir la estructura factorial de la escala, se efectuaron dos análisis de componentes principales con rotación Varimax, con criterio Kaiser de autovalores mayores que 1 (Hair et al., 1999). El primero incluyó las 22 escalas y el segundo, las 11 escalas clínicas.
Tras ello, se llevó a cabo el análisis Procustes sin ajuste (también llamado no-Procustes análisis), mediante el software Orthosim (Barrett, 2005; Hoelzle y Meyer, 2009), para examinar la congruencia de los componentes analizados en este estudio $\mathrm{y}$ en las versiones para adultos alemana, estadounidense y española. El programa presenta dos restricciones en cuanto al análisis de los datos. En primer lugar, la cantidad de variables en la matriz-objetivo no puede ser inferior a la de la matriz de comparación -por lo que no se pudo comparar la versión de cinco factores española con la que se detallará en este trabajo, en la que se hallaron cuatro dimensiones-. En segundo lugar, deben utilizarse todas las saturaciones factoriales y dado que los autores chinos solo reportaron los valores de las cargas superiores a 0,30 , no se pueden efectuar comparaciones con esa versión.

\section{Resultados}

Como primer paso, se calcularon estadísticos descriptivos (media, desvío, asimetría) para escalas y subescalas. Como se detalla en la tabla 2, las medias resultaron bajas y los valores de asimetría fueron menores que 2 , a excepción de la escala de ideación suicida.

\section{Fiabilidad}

En la tabla 3, se informan las alfas de Cronbach, que se ubicaron en el rango entre 0,62 y 0,81 para las escalas. Los valores más bajos correspondieron a dos de las escalas de validez: impresión positiva $(0,64)$ e impresión negativa $(0,65)$. También se hallaron guarismos

Tabla 2

Estadísticos descriptivos internos para escalas y subescalas del Inventario de Evaluación de la Personalidad para adolescentes

\begin{tabular}{|c|c|c|c|c|c|c|c|c|c|}
\hline Escalas & $\begin{array}{c}\text { Máximo } \\
\text { posible }\end{array}$ & $M$ & $D E$ & Asimetría & Subescalas & $\begin{array}{c}\text { Máximo } \\
\text { posible }\end{array}$ & $M$ & $D E$ & Asimetría \\
\hline INC & 30 & 4,26 & 2,83 & 0,93 & - & - & - & - & - \\
\hline INF & 21 & 3,70 & 2,39 & 0,68 & - & - & - & - & - \\
\hline IMN & 24 & 2,29 & 2,88 & 1,88 & - & - & - & - & - \\
\hline IMP & 18 & 8,75 & 3,43 & $-0,08$ & - & - & - & - & - \\
\hline \multirow{3}{*}{ SOM } & \multirow{3}{*}{54} & \multirow{3}{*}{7,05} & \multirow{3}{*}{6,17} & \multirow{3}{*}{1,43} & SOM-C & 18 & 2,49 & 2,75 & 1,44 \\
\hline & & & & & SOM-S & 18 & 2,49 & 2,62 & 1,23 \\
\hline & & & & & SOM-H & 18 & 2,06 & 2,34 & 1,97 \\
\hline
\end{tabular}


(viene)

\begin{tabular}{|c|c|c|c|c|c|c|c|c|c|}
\hline Escalas & $\begin{array}{c}\text { Máximo } \\
\text { posible }\end{array}$ & $M$ & $D E$ & Asimetría & Subescalas & $\begin{array}{c}\text { Máximo } \\
\text { posible }\end{array}$ & $M$ & $D E$ & Asimetría \\
\hline \multirow{3}{*}{ ANS } & \multirow{3}{*}{54} & \multirow{3}{*}{16,10} & \multirow{3}{*}{7,56} & \multirow{3}{*}{0,74} & ANS-C & 18 & 4,28 & 3,25 & 0,85 \\
\hline & & & & & ANS-E & 18 & 7,29 & 3,06 & 0,33 \\
\hline & & & & & ANS-F & 18 & 4,53 & 2,82 & 0,81 \\
\hline \multirow{3}{*}{ TRA } & \multirow{3}{*}{54} & \multirow{3}{*}{17,07} & \multirow{3}{*}{6,67} & \multirow{3}{*}{0,55} & TRA-O & 18 & 5,57 & 3,09 & 0,50 \\
\hline & & & & & TRA-F & 18 & 7,51 & 2,95 & 0,24 \\
\hline & & & & & TRA-E & 18 & 3,98 & 3,81 & 1,01 \\
\hline \multirow{3}{*}{ DEP } & \multirow{3}{*}{54} & \multirow{3}{*}{13,47} & \multirow{3}{*}{6,60} & \multirow{3}{*}{0,85} & DEP-C & 18 & 4,83 & 2,75 & 0,81 \\
\hline & & & & & DEP-E & 18 & 3,54 & 2,69 & 1,20 \\
\hline & & & & & DEP-F & 18 & 5,09 & 2,95 & 0,33 \\
\hline \multirow{3}{*}{ MAN } & \multirow{3}{*}{54} & \multirow{3}{*}{19,91} & \multirow{3}{*}{7,77} & \multirow{3}{*}{0,25} & MAN-A & 18 & 4,57 & 3,01 & 0,59 \\
\hline & & & & & MAN-G & 18 & 7,22 & 3,43 & 0,34 \\
\hline & & & & & MAN-I & 18 & 8,11 & 4,02 & 0,17 \\
\hline \multirow{3}{*}{ PAR } & \multirow{3}{*}{54} & \multirow{3}{*}{17,97} & \multirow{3}{*}{6,95} & \multirow{3}{*}{0,71} & PAR-H & 18 & 7,36 & 3,25 & 0,39 \\
\hline & & & & & PAR-P & 18 & 2,44 & 2,89 & 1,54 \\
\hline & & & & & PAR-R & 18 & 8,17 & 3,16 & 0,13 \\
\hline \multirow{3}{*}{ ESQ } & & & & & ESQ-A & 18 & 3,11 & 2,84 & 1,23 \\
\hline & 54 & 11,51 & 6,92 & 0,78 & ESQ-P & 18 & 4,14 & 2,95 & 0,71 \\
\hline & & & & & ESQ-S & 18 & 4,26 & 3,52 & 0,74 \\
\hline & & & & & LIM-A & 15 & 5,47 & 2,78 & 0,48 \\
\hline IIM & 60 & 2051 & 0 & 053 & LIM-E & 15 & 6,32 & 2,83 & 0,41 \\
\hline $\mathrm{LIM}$ & 00 & 20,51 & 9,20 & 0,53 & LIM-I & 15 & 4,92 & 3,43 & 0,54 \\
\hline & & & & & LIM-P & 15 & 3,80 & 2,84 & 0,67 \\
\hline & & & & & ANT-A & 24 & 4,03 & 3,30 & 0,83 \\
\hline ANT & 54 & 13,14 & 7,54 & 0,79 & ANT-E & 24 & 3,29 & 2,87 & 1,05 \\
\hline & & & & & ANT-B & 24 & 5,82 & 3,14 & 0,60 \\
\hline ALC & 24 & 2,82 & 3,34 & 1,84 & - & - & & & \\
\hline DRG & 24 & 2,21 & 3,41 & 1,87 & - & - & & & \\
\hline & & & & & AGR - A & 18 & 7,37 & 3,40 & 0,18 \\
\hline AGR & 54 & 19,55 & 8,49 & 0,53 & AGR $-\mathrm{V}$ & 18 & 8,73 & 3,68 & 0,12 \\
\hline & & & & & $\mathrm{AGR}-\mathrm{F}$ & 18 & 3,45 & 3,33 & 1,14 \\
\hline SUI & 24 & 1,75 & 3,27 & 2,84 & - & - & - & - & - \\
\hline EST & 18 & 3,10 & 3,15 & 1,21 & - & - & - & - & - \\
\hline FAS & 18 & 4,27 & 3,31 & 0,69 & - & - & - & - & - \\
\hline RTR & 18 & 10,91 & 3,64 & $-0,32$ & - & - & - & - & - \\
\hline DOM & 24 & 12,56 & 3,97 & 0,26 & - & - & - & - & - \\
\hline AFA & 24 & 14,48 & 4,26 & $-0,22$ & - & - & - & - & - \\
\hline
\end{tabular}

Nota . INC $=$ Inconsistencia, $\mathrm{INF}=$ Infrecuencia, $\mathrm{IMN}=$ Impresión negativa, $\mathrm{IMP}=$ Impresión positiva, sOM=Somatizaciones, ANS $=$ Ansiedad, TRA $=$ Trastornos relacionados con la ansiedad, DEP $=$ Depresión, $\mathrm{MAN}=$ Manía, PAR $=$ Paranoia, ESQ $=$ Esquizofrenia, LIM = Rasgos límites, ANT = Rasgos antisociales, ALC = Problemas con el alcohol, DRG = Problemas con las drogas, $\mathrm{AGR}=$ Agresión, $\mathrm{SUI}=$ Ideación suicida, $\mathrm{EST}=$ Estrés, FAS = Falta de apoyo social, $\mathrm{RTR}=$ Rechazo al tratamiento, $\mathrm{DOM}=$ Dominancia, $\mathrm{AFA}=$ Afabilidad . Elaboración propia 
inferiores en dominancia $(0,62)$ y falta de apoyo social $(0,65)$. Los más elevados se obtuvieron para rasgos límites $(0,81)$, agresión $(0,80)$ e ideación suicida $(0,80)$.

En las subescalas, los valores fueron más bajos, ubicados entre 0,33 y 0,79, con la mayoría entre 0,50 y 0,79 . Las alfas con menores valores se presentaron en trastornos relacionados con la ansiedad/obsesivo-compulsivo $(0,33)$, rasgos límite/problemas de identidad $(0,45)$, ansiedad/fisiológica $(0,47)$ y depresión/fisiológica $(0,47)$; mientras que los más elevados se presentaron en trastornos relacionados con la ansiedad/estrés postraumático $(0,79)$ y paranoia/persecución $(0,75)$.

Tabla 3

Consistencia interna para escalas y subescalas del Inventario de Evaluación de la Personalidad para adolescentes

\begin{tabular}{|c|c|c|c|c|c|}
\hline Escalas & Alfa de Cronbach & $\begin{array}{c}\text { Promedio de } \\
\text { correlaciones } \\
\text { inter-ítem }\end{array}$ & Subescalas & Alfa de Cronbach & $\begin{array}{c}\text { Promedio de } \\
\text { correlaciones } \\
\text { inter-ítem }\end{array}$ \\
\hline IMN & 0,65 & 0,19 & - & - & - \\
\hline IMP & 0,64 & 0,23 & - & - & - \\
\hline \multirow{3}{*}{ SOM } & \multirow{3}{*}{0,77} & \multirow{3}{*}{0,17} & SOM-C & 0,62 & 0,22 \\
\hline & & & SOM-S & 0,53 & 0,16 \\
\hline & & & SOM-H & 0,58 & 0,24 \\
\hline \multirow{3}{*}{ ANS } & \multirow{3}{*}{0,79} & \multirow{3}{*}{0,18} & ANS-C & 0,68 & 0,26 \\
\hline & & & ANS-E & 0,57 & 0,19 \\
\hline & & & ANS-F & 0,48 & 0,15 \\
\hline \multirow{3}{*}{ TRA } & \multirow{3}{*}{0,66} & \multirow{3}{*}{0,10} & TRA-O & 0,47 & 0,13 \\
\hline & & & TRA-F & 0,33 & 0,08 \\
\hline & & & TRA-E & 0,79 & 0,38 \\
\hline \multirow{3}{*}{ DEP } & \multirow{3}{*}{0,74} & \multirow{3}{*}{0,15} & DEP-C & 0,53 & 0,16 \\
\hline & & & DEP-E & 0,58 & 0,22 \\
\hline & & & DEP-F & 0,48 & 0,12 \\
\hline \multirow{3}{*}{ MAN } & \multirow{3}{*}{0,75} & \multirow{3}{*}{0,14} & MAN-A & 0,49 & 0,14 \\
\hline & & & MAN-G & 0,61 & 0,21 \\
\hline & & & MAN-I & 0,73 & 0,31 \\
\hline \multirow{3}{*}{ PAR } & \multirow{3}{*}{0,75} & \multirow{3}{*}{0,15} & PAR-H & 0,59 & 0,19 \\
\hline & & & PAR-P & 0,75 & 0,33 \\
\hline & & & PAR-R & 0,52 & 0,16 \\
\hline \multirow{3}{*}{ ESQ } & \multirow{3}{*}{0,77} & \multirow{3}{*}{0,16} & ESQ-A & 0,62 & 0,31 \\
\hline & & & ESQ-P & 0,62 & 0,22 \\
\hline & & & ESQ-S & 0,62 & 0,21 \\
\hline \multirow{4}{*}{ LIM } & \multirow{4}{*}{0,81} & \multirow{4}{*}{0,18} & LIM-A & 0,60 & 0,23 \\
\hline & & & LIM-E & 0,50 & 0,16 \\
\hline & & & LIM-I & 0,45 & 0,14 \\
\hline & & & LIM-P & 0,65 & 0,29 \\
\hline \multirow{3}{*}{ ANT } & \multirow{3}{*}{0,76} & \multirow{3}{*}{0,16} & ANT-A & 0,58 & 0,19 \\
\hline & & & ANT-E & 0,57 & 0,18 \\
\hline & & & ANT-B & 0,51 & 0,16 \\
\hline
\end{tabular}




\begin{tabular}{|c|c|c|c|c|c|}
\hline Escalas & Alfa de Cronbach & $\begin{array}{l}\text { Promedio de } \\
\text { correlaciones } \\
\text { inter-ítem }\end{array}$ & Subescalas & Alfa de Cronbach & $\begin{array}{c}\text { Promedio de } \\
\text { correlaciones } \\
\text { inter-ítem }\end{array}$ \\
\hline ALC & 0,72 & 0,29 & - & & \\
\hline \multirow[t]{2}{*}{ DRG } & 0,69 & 0,28 & - & & \\
\hline & & & AGR -A & 0,58 & 0,19 \\
\hline \multirow[t]{2}{*}{ AGR } & 0,80 & 0,20 & AGR $-\mathrm{V}$ & 0,61 & 0,21 \\
\hline & & & AGR -F & 0,67 & 0,29 \\
\hline SUI & 0,80 & 0,42 & - & - & - \\
\hline EST & 0,68 & 0,27 & - & - & - \\
\hline FAS & 0,65 & 0,24 & - & - & - \\
\hline RTR & 0,70 & 0,28 & - & - & - \\
\hline DOM & 0,62 & 0,16 & - & - & - \\
\hline AFA & 0,69 & 0,23 & - & - & - \\
\hline
\end{tabular}

Nota INC $=$ Inconsistencia, $\mathrm{INF}=$ Infrecuencia, $\mathrm{IMN}=$ Impresión negativa, $\mathrm{IMP}=$ Impresión positiva, soM $=$ Somatizaciones, ANS $=$ Ansiedad, TRA $=$ Trastornos relacionados con la ansiedad, DEP $=$ Depresión, MAN=Manía, PAR = Paranoia, ESQ $=$ Esquizofrenia, LIM = Rasgos límites, ANT = Rasgos antisociales, ALC = Problemas con el alcohol, DRG = Problemas con las drogas, AGR = Agresión, sUI = Ideación suicida, EST = Estrés, FAS = Falta de apoyo social, $\mathrm{RTR}=$ Rechazo al tratamiento, $\mathrm{DOM}=$ Dominancia, $\mathrm{AFA}=$ Afabilidad . Elaboración propia

\section{Estructura factorial}

Al efectuar el análisis de componentes principales con las 22 escalas, se halló una solución factorial de cuatro dimensiones que explicó el $62,31 \%$ de la varianza ( $\mathrm{kм}=0,929$; Bartlet: $\mathrm{X}^{2}=11870,68 ; \mathrm{gl}=231$, $\mathrm{p}=<0,001)$. El primer factor incluyó con cargas positivas a las escalas de impresión negativa, inconsistencia, quejas somáticas, ansiedad, trastornos relacionados con la ansiedad, depresión, manía, paranoia, esquizofrenia, rasgos antisociales, ideación suicida y estrés; mientras que infrecuencia y trastornos relacionados con el tratamiento presentaron valores negativos. El segundo factor agrupó a las escalas de impresión negativa, inconsistencia, rasgos antisociales, problemas con el alcohol, problemas con las drogas, ideación suicida y estrés; el tercero, a impresión positiva, depresión, falta de apoyo social y afabilidad; y el cuarto, a manía, rasgos antisociales, agresión y dominancia.

En el análisis de componentes principales realizado con las 11 escalas clínicas, se aisló una estructura factorial bidimensional que explicaba el 60,78\% de la varianza $\left(\mathrm{Kмо}=0,88\right.$; Bartlet: $\mathrm{X}^{2}=5395,96$; $\mathrm{gl}=55 ; \mathrm{p}=<0,001)$. La primera dimensión incluyó las escalas de quejas somáticas, ansiedad, trastornos relacionados con la ansiedad, depresión, manía, paranoia, esquizofrenia, rasgos límites y rasgos antisociales; la segunda, a rasgos antisociales, problemas con el alcohol y problemas con las drogas (tabla 4).

Al comparar las estructuras factoriales de este estudio con los resultados alemanes para las 22 escalas, se encontró una congruencia global moderada $(0,84)$, y sucedió lo mismo en los tres primeros factores, dado que sus valores se ubicaron entre 0,82 y 0,92 (Barrett, 2005; Hoelzle y Meyer, 2009). En el caso del cuarto factor, la congruencia fue baja (tabla 5).

$\mathrm{Al}$ analizar la congruencia de las soluciones factoriales de dos dimensiones halladas para las 11 escalas en función de los resultados alemanes y estadounidenses, se observó una congruencia moderada tanto en los coeficientes globales como en los factores, a excepción del segundo factor del estudio de Morey (2007b), en el que los valores fueron bajos. En relación con los hallazgos españoles, se advirtió una congruencia global buena $(0,93)$, al igual que para el primer factor $(0,95)$, aunque moderada en el segundo $(0,86)$. 
Tabla 4

Cargas de los ítems y factores extraídos para el Inventario de Evaluación de la Personalidad para adolescentes

\begin{tabular}{|c|c|c|c|c|c|c|}
\hline \multirow{2}{*}{ Escalas } & \multicolumn{4}{|c|}{22 Escalas } & \multicolumn{2}{|c|}{11 Escalas } \\
\hline & F1 & F2 & F3 & F4 & F1 & $\mathrm{F} 2$ \\
\hline IMN & 0,44 & 0,53 & 0,21 & 0,15 & & \\
\hline IMP & $-0,01$ & 0,22 & 0,59 & 0,11 & & \\
\hline INC & 0,60 & 0,48 & 0,25 & 0,09 & & \\
\hline INF & $-0,70$ & 0,11 & 0,05 & $-0,31$ & & \\
\hline SOM & 0,58 & 0,46 & 0,14 & 0,04 & 0,63 & 0,38 \\
\hline ANS & 0,85 & 0,09 & 0,11 & $-0,01$ & 0,84 & 0,04 \\
\hline TRA & 0,75 & 0,10 & 0,01 & 0,01 & 0,78 & $-0,02$ \\
\hline DEP & 0,63 & 0,28 & 0,47 & $-0,13$ & 0,67 & 0,32 \\
\hline MAN & 0,48 & 0,01 & $-0,11$ & 0,66 & 0,62 & $-0,01$ \\
\hline PAR & 0,63 & 0,16 & 0,38 & 0,32 & 0,76 & 0,25 \\
\hline ESQ & 0,66 & 0,25 & 0,38 & 0,09 & 0,76 & 0,29 \\
\hline LIM & 0,79 & 0,21 & 0,10 & 0,30 & 0,81 & 0,25 \\
\hline ANT & 0,32 & 0,42 & 0,24 & 0,54 & 0,46 & 0,56 \\
\hline ALC & 0,09 & 0,76 & 0,05 & 0,08 & 0,09 & 0,82 \\
\hline DRG & $-0,03$ & 0,77 & 0,14 & 0,07 & 0,03 & 0,81 \\
\hline AGR & 0,38 & 0,10 & 0,36 & 0,62 & & \\
\hline SUI & 0,42 & 0,59 & 0,28 & $-0,03$ & & \\
\hline EST & 0,54 & 0,40 & 0,19 & 0,08 & & \\
\hline FAS & 0,29 & 0,26 & 0,64 & 0,07 & & \\
\hline RTR & $-0,78$ & $-0,15$ & 0,14 & $-0,07$ & & \\
\hline DOM & $-0,23$ & 0,08 & $-0,24$ & 0,70 & & \\
\hline AFA & 0,08 & $-0,01$ & $-0,78$ & 0,26 & & \\
\hline \% Varianza explicada & 28,33 & 13,51 & 11,10 & 9,35 & 41,61 & 19,16 \\
\hline
\end{tabular}

Nota . INC = Inconsistencia, $\mathrm{INF}=$ Infrecuencia, $\mathrm{IMN}=$ Impresión negativa, $\mathrm{IMP}=$ Impresión positiva, SOM $=$ Somatizaciones, ANS $=$ Ansiedad, TRA $=$ Trastornos relacionados con la ansiedad, DEP $=$ Depresión, MAN $=$ Manía, PAR $=$ Paranoia, ESQ = Esquizofrenia, LIM $=$ Rasgos lí mites, $\mathrm{ANT}=$ Rasgos antisociales, $\mathrm{ALC}=$ Problemas con el alcohol, $\mathrm{DRG}=$ Problemas con las drogas, $\mathrm{AGR}=$ Agresión, $\mathrm{sUI}=\mathrm{Ideación}$ suicida , EST $=$ Estrés, FAS $=$ Falta de apoyo social, $\mathrm{RTR}=$ Rechazo al tratamiento, $\mathrm{DOM}=$ Dominancia, $\mathrm{AFA}=$ Afabilidad . Elaboración propia

Tabla 5

Comparación de estructuras factoriales - coeficientes de congruencia.

\begin{tabular}{ccccc}
\hline Escalas & 22 & \multicolumn{3}{c}{11} \\
Factores & 4 & & 2 & \\
Versión & Alemania & EE. UU. & España & Alemania \\
\hline Global & 0,84 & 0,91 & 0,93 & 0,91 \\
$\mathbf{1}$ & 0,89 & 0,91 & 0,95 & 0,91 \\
$\mathbf{2}$ & 0,90 & 0,65 & 0,86 & 0,86 \\
$\mathbf{3}$ & 0,84 & - & - & - \\
4 & 0,79 & - & - & - \\
\hline
\end{tabular}

Nota. Elaboración propia

\section{Discusión}

El PAI-A se presenta como un instrumento de interés considerando lo reciente de las investigaciones acerca de él (Morey y Meyer, 2013) y la evidencia sobre su calidad psicométrica (Cheung et al., 2008; Morey, 2007a). Por lo tanto, en el presente artículo se hicieron análisis con una muestra de adolescentes de la ciudad de Buenos Aires y alrededores, con el objetivo de contar con un instrumento de evaluación diagnóstica adaptado a las peculiaridades de esa población. 
Tras revisar los aspectos lingüísticos, se estimó la consistencia interna, con lo que se encontraron alfas de Cronbach adecuadas para las escalas clínicas y relacionadas con el tratamiento, mientras que en las escalas de validez y subescalas resultaron más bajos, pero aceptables en el marco del ámbito de investigación. Estos resultados son similares a los reportados en investigaciones previas (Cheung et al., 2008; Groves y Engel, 2007; Lyrakos, 2011; Morey, 2007a y 2007b; Ortiz-Tallo et al., 2011), y a la vez presentan coherencia con la cantidad de ítems incluidos en los análisis: las escalas se componen de mayor número de reactivos y las subescalas, de uno menor. Los hallazgos aquí expuestos dan cuenta de un aspecto vinculado a la confiabilidad de las puntuaciones del instrumento.

Respecto a la dimensionalidad, en el análisis de componentes principales que incluyó las 22 escalas se aislaron cuatro factores, al igual que en las adaptaciones anteriores llevadas a cabo con adultos alemanes (Groves y Engel, 2007) y adolescentes chinos (Cheung et al., 2008). La primera dimensión agrupó una amplia gama de sintomatologías, sobre todo internalizante (quejas somáticas, ansiedad, trastornos relacionados con la ansiedad, depresión, ideación suicida, estrés) y relativa a alteraciones del pensamiento (paranoia, esquizofrenia) y a problemas del control de los impulsos (manía, rasgos antisociales). Estos síntomas se corresponden con los primeros tres agrupamientos sugeridos por Blais et al. (2011) al momento de interpretar los perfiles del PAI-A. Así mismo, en este factor se incluyeron: impresión negativa e inconsistencia con cargas factoriales positivas, y rechazo al tratamiento con saturaciones negativas. Eso parece lógico si se tiene en cuenta que este factor intenta representar el malestar que el sujeto experimenta, así como una posible dificultad para responder de modo completamente consistente. Las cargas negativas detectadas en infrecuencia parecen coherentes considerando que evalúan respuestas poco frecuentes y que se trata de una muestra de población general.

El segundo factor reunió sintomatologías de carácter externalizante (rasgos antisociales, problemas con el alcohol, problemas con las drogas), vinculadas al segundo grupo propuesto por Blais et al. (2011), así como ideación suicida y estrés. También se presentaron impresión negativa e inconsistencia, con lo que pudieron hipotetizarse explicaciones similares a las mencionadas en el párrafo anterior para el primer factor. En el tercer factor, se reúnen manifestaciones disfóricas (depresión, falta de apoyo social) y rasgos de sociabilidad (impresión positiva, afabilidad). Por último, en la cuarta dimensión nuevamente aparecen síntomas externalizantes (manía, rasgos antisociales, agresión, dominancia).

En el análisis de componentes principales realizado con las 11 escalas clínicas, se aisló una estructura factorial de dos dimensiones, al igual que en las versiones para adultos estadounidense (Morey, 2007b), alemana (Groves y Engel, 2007) y española (Ortiz-Tallo et al., 2011), y en los estudios con adolescentes chinos (Cheung et al., 2008).

De modo similar al análisis con las 22 escalas, en el primer factor se agruparon síntomas vinculados a la ansiedad (quejas somáticas, ansiedad, trastornos relacionados con la ansiedad, depresión) y a perturbaciones del pensamiento (paranoia, esquizofrenia) e impulsividad (manía, rasgos límites, rasgos antisociales); mientras que el segundo factor resulta similar al segundo agrupamiento de patrones externalizantes (rasgos antisociales, problemas con el alcohol y problemas con las drogas) propuesto por Blais et al. (2011).

La varianza explicada en ambos análisis fue adecuada, resultando superior al $60 \%$. Al comparar las estructuras factoriales halladas en esta investigación con los análisis estadounidenses y alemanes, se detectó una congruencia moderada en la mayoría de los coeficientes. Al contrastar con los resultados españoles, apareció una mayor adecuación.

Dos aspectos deben analizarse en relación con estos hallazgos. En primer lugar, la cantidad de factores aislados resulta similar a la de investigaciones previas: se encontraron cuatro al incluir las 22 escalas, tal como en las adaptaciones alemana y china, y dos al examinar solamente las 11 escalas clínicas, igual número que el obtenido en los estudios con población estadounidense, alemana, española y china. Estos resultados dan cuenta de una estabilidad del agrupamiento de los patrones sintomáticos a través de varias culturas.

En segundo lugar, debe mencionarse que dichas estructuras no son completamente compatibles, dado que los coeficientes de congruencia han sido en su mayoría moderados. La excepción se presentó al comparar con la versión para adultos española, encontrándose valores superiores. Esta mayor semejanza puede interpretarse en función de las similitudes en los reactivos (dado que la versión argentina parte de la versión española), y en función de los parecidos idiomáticos y culturales.

Para considerar las limitaciones de este estudio, si bien los análisis de componentes principales se efectuaron con el objetivo de replicar investigaciones precedentes, utilizar las puntuaciones totales 
como input constituye una debilidad metodológica que debe ser mejorada en futuros trabajos en los que se empleen los ítems como input. Se sugiere que futuras líneas de investigación realicen análisis factoriales exploratorios cuyo input sean los ítems.

Otro aspecto a señalar lo constituye el hecho de que no se eliminaran protocolos de acuerdo con las escalas de validez, debido a que aún no se dispone de baremos locales. Sin embargo, debe resaltarse que al examinar las medias y los desvíos de las escalas de validez, los valores fueron bajos, lo cual puede interpretarse como un indicador de validez de los protocolos.

Por último, la muestra se circunscribió a adolescentes escolarizados de la ciudad de Buenos Aires y alrededores, por lo cual los resultados solo pueden generalizarse a sujetos con similares características sociodemográficas, siendo de interés que en futuros trabajos se continúe esta línea de investigación replicando los análisis en población clínica local adolescente.

\section{Conclusiones}

Para finalizar, se destaca que el trabajo aquí expuesto aporta evidencias sobre la calidad psicométrica del PAI-A para su uso en población de Buenos Aires. Esto resulta relevante en función de la importancia que las manifestaciones de trastornos mentales presentan en los adolescentes (oMs, 2014).

Lacorrecta evaluación dela psicopatología con un instrumento válido y confiable permitirá no solo llegar a un diagnóstico preciso mediante el PAI-A (considerando sus escalas clínicas), sino también diseñar un tratamiento acorde a las características del evaluado y su entorno (de acuerdo con las escalas relacionadas con el tratamiento y la relación interpersonal).

Enconclusión, seaportauninstrumentoquecubre un área de vacancia local debido al envejecimiento de los instrumentos de evaluación actualmente disponibles, lo cual permitirá el trabajo con los adolescentes, siendo el grupo etario con mayor riesgo de desarrollar psicopatología (Casullo y Fernández Liporace, 2001; Vega et al., 2009), la cual repercute tanto en su calidad de vida actual como en su futuro (Helgeland et al., 2005; Quiroga et al., 2013; Stepp et al., 2013).

\section{Referencias}

American Psychiatric Association. (2013). Diagnostic and Statistical Manual of Mental Disorders ( $5^{\text {th }}$ edition). Washington: American Psychiatric Association.
Barrett, P. T. (2005). Orthosim 2 (version 2.0): Target-comparison matrix fitting [Computer software and manual]. Recuperado de https://goo.gl/LrbMGr

Blais, M. A., Baity, M. R. y Hopwood, C. J. (2011). Clinical applications of the Personality Assessment Inventory. Londres: Routledge.

Busch, A., Morey, L. C. y Hopwood, C. J. (2017). Exploring the assessment of the DSM- 5 alternative model for personality disorders with the Personality Assessment Inventory. Journal of Personality Assessment, 99, 211218. doi: $10.1080 / 00223891.2016 .1217872$

Cardenal, V., Ortiz-Tallo, M. y Santamaría, P. (2012). PAI-A. Inventario de evaluación de la personalidad para adolescentes. Manual de aplicación, versión experimental. Madrid: TEA Ediciones.

Casullo, M. M. (2003). Adaptación del Inventario MMPI-A. Buenos Aires: Universidad de Buenos Aires.

Casullo, M. M. y Fernández Liporace, M. (2001). Malestares psicológicos en estudiantes adolescentes argentinos. Psykhe, 10(1), 155-162.

Casullo, M. M., Góngora, V. y Castro, A. (1998). La adaptación del inventario MACI (Millon Adolescent Clinical Inventory). Un estudio preliminar con estudiantes adolescentes argentinos. Investigaciones en Psicología, 3(2), 73-89.

Casullo, M. M. y Pérez, M. (1998). El listado de síntomas SCL-90-R de Derogatis. Buenos Aires: Universidad de Buenos Aires.

Cheung, F. M., Fan, W., Cheung, S. F. y Leung, K. (2008). Standarization of the Cross-Cultural (Chinese) Personality Assessment Inventory for Adolescents in Hong Kong: A combined emic-etic approach to personality assessment. Acta Psychologica Sinica, 40(7), 839-852.

Desuque, D. A., Rubilar, J. V. y Lemos, V. N. (2011). Análisis psicométrico del Cuestionario de Creencias Actitudinales sobre el Comportamiento Suicida en población adolescente en Entre Ríos, Argentina. Liberabit, 17(2), 187-198.

Fernández Liporace, M. y Casullo, M. M. (2006). Validación factorial de una escala para evaluar riesgo suicida. Revista Iberoamericana de Evaluación Psicológica, 21(1), 9-22.

Góngora, V. A. (2010). Evaluación de la estructura factorial de la prueba MAC-R (Cuestionario de Cogniciones Anoréxicas de Mizes) en población femenina adulta y adolescente de la ciudad de Buenos Aires. Revista Iberoamericana de Evaluación Psicológica, 30(2), 71-86.

Grañana, N., Richaudeau, A., Robles Gorriti, C., O’Flaherty, M., Scotti, M. E., Sixto, L., et al. (2011). Evaluación de déficit de atención con hiperactividad: la escala SNAP IV adaptada a la Argentina. Revista Panamericana de Salud Pública, 29(5), 344-349. 
Groves, J. A. y Engel, R. R. (2007). The German adaptation and standardization of the Personality Assessment Inventory (PAI). Journal of Personality Assessment, 88(1), 49-56.

Hair, J. F., Anderson, R. E., Tatham, R. L. y Black, W. C. (1999). Análisis multivariante. Madrid: Prentice Hall.

Hambleton, R. K. y Zenisky, A. L. (2011). Translating and adapting tests for cross-cultural assessments. En D. Matsumoto y F. J. R. Van de Vijver (Eds.), Cross-cultural research methods in psychology (pp. 46-70). Nueva York: Cambridge University Press.

Helgeland, M. I., Kjelsberg, E. y Torgersen, S. (2005). Continuities between emotional and disruptive behavior disorders in adolescence and personality disorders in adulthood. American Journal of Psychiatry, 162, 19411947. doi: 10.1176/appi.ajp.162.10.1941

Ho, M. Y., Cheung, F. M. y Cheung, S. F. (2008). Personality and life events as predictors of adolescents' life satisfaction: Do life events mediate the link between personality and life satisfaction? Social Indicators Research, 89(3), 457-471. doi: 10.1007/s11205-008-9243-6

Hoelzle, J. B. y Meyer, G. J. (2009). The invariant component structure of Personality Assessment Inventory (PAI) full scales. Journal of Personality Assessment, 91(2), 175-186. doi: 10.1080/00223890802634316

Hopwood, C. J., Wright, A. G. C., Krueger, R. F., Schade, N., Markon, K. E. y Morey, L. C. (2013). Dsm-5 pathological personality traits and the Personality Assessment Inventory. Assessment, 20(3), 269-285. doi: $10.1177 / 1073191113486286$

Lyrakos, D. G. (2011). The development of the Greek Personality Assessment Inventory. Psychology, 2(8), $797-$ 803. doi: 10.4236/psych.2011.28122

Morey, L. (1991). Personality Assessment Inventory professional manual. Odessa: Psychological Assessment Resources.

Morey, L. (2007a). Personality Assessment Inventory Adolescent Professional Manual. Lutz: Psychological Assessment Resources.

Morey, L. (2007b). Personality Assessment Inventory (PAI). Professional Manual ( $2^{\text {nd }}$ edition). Odessa: Psychological Assessment Resources.

Morey, L. C. y Meyer, J. K. (2013). Forensic assessment with the Personality Assessment Inventory. En R. Archer y E. Wheeler (Eds.), Forensic uses of clinical assessment instruments (pp. 140-176). Londres: Routledge.
Morey, L. C. y Meyer, J. K. (2014). Conceptualizing youth borderline personality disorder within a PAI Framework. En C. Sharp (Ed.), Handbook of Borderline Personality Disorder in Children and Adolescents (pp. 49-65). Nueva York: Springer.

Muñiz, J., Elosua, P. y Hambleton, R. K. (2013). Directrices para la traducción y adaptación de los tests: segunda edición. Psicothema, 25(2), 151-157. doi: 10.7334/psicothema201

Oros, L. B. y Neifert, I. (2006). Construcción y validación de una escala para evaluar indicadores físicos y psicoemocionales de estrés. Evaluar, 6, 1-14.

Ortiz-Tallo, M., Santamaría, P., Cardenal, V. y Sánchez, M. P. (2011). Inventario de evaluación de la personalidad. Madrid: TEA Ediciones.

Quiroga, C. V., Janosz, M., Bisset, S. y Morin, A. J. S. (2013). Early adolescent depression symptoms and school dropout: Mediating processes involving self-reported academic competence and achievement. Journal of Educational Psychology, 105(2), 552-560. doi: 10.1037/ a0031524

Ríos, J. y Morey, L. C. (2013). Detecting feigned ADHD in later adolescence: An examination of three PAI-A negative distortion indicators. Journal of Personality Assessment, 95(6), 594-599. doi 10.1080/00223891.2013.821071

Rutsztein, G., Leonardelli, E., Scappatura, M. L., Murawski, B., Elizathe, L. y Maglio, A. L. (2013). Propiedades psicométricas del Inventario de Trastornos Alimentarios - 3 (EDI-3) en mujeres adolescentes de Argentina. Revista Mexicana de Trastornos Alimentarios, 4, 1-14.

Stepp, S. D., Olino, T. M., Klein, D. N., Seeley, J. R. y Lewinsohn, P. M. (2013). Unique influences of adolescent antecedents on adult borderline personality disorder features. Personality Disorders, 4(3), 223-229. doi: 10.1037/per0000015

Turner, E. A. (2014). Use of the PAI-A in the diagnosis and treatment of psychiatric disorders: A clinical application. Annals of Psychiatry and Mental Health, 2(1), 1-5.

Vega, V. C., Piccini, M., Barrionuevo, J. A. y Tocci, R. F. (2009). Depresión y trastornos de la conducta alimentaria en una muestra no clínica de adolescentes mujeres. Anuario de Investigaciones, 16, 103-114.

World Health Organization. (2014). Health for the world's adolescents. Génova: World Health Organization. 